\title{
Existence and Global Exponential Stability of Almost Periodic Solutions for SICNNs with Nonlinear Behaved Functions and Mixed Delays
}

\author{
Xinsong Yang, ${ }^{1}$ Jinde Cao, ${ }^{2}$ Chuangxia Huang, ${ }^{3}$ and Yao Long ${ }^{1}$ \\ ${ }^{1}$ Department of Mathematics, Honghe University, Mengzi Yunnan 661100, China \\ 2 Department of Mathematics, Southeast University, Nanjing 210096, China \\ ${ }^{3}$ Department of Mathematics, College of Mathematics and Computing Science, \\ Changsha University of Science and Technology, Changsha, Hunan 410076, China
}

Correspondence should be addressed to Jinde Cao, jdcaoseu@gmail.com

Received 9 July 2009; Accepted 2 February 2010

Academic Editor: Allan C. Peterson

Copyright (c) 2010 Xinsong Yang et al. This is an open access article distributed under the Creative Commons Attribution License, which permits unrestricted use, distribution, and reproduction in any medium, provided the original work is properly cited.

By using the Leray-Schauder fixed point theorem and differential inequality techniques, several new sufficient conditions are obtained for the existence and global exponential stability of almost periodic solutions for shunting inhibitory cellular neural networks with discrete and distributed delays. The model in this paper possesses two characters: nonlinear behaved functions and all coefficients are time varying. Hence, our model is general and applicable to many known models. Moreover, our main results are also general and can be easily deduced to many simple cases, including some existing results. An example and its simulation are employed to illustrate our feasible results.

\section{Introduction}

Consider the following shunting inhibitory cellular neural networks (SICNNs) with discrete and distributed delays (mixed delays):

$$
\begin{aligned}
x_{i j}^{\prime}(t)= & -a_{i j}\left(t, x_{i j}(t)\right)+\sum_{B^{k l} \in N_{r}(i, j)} B_{i j}^{k l}(t) f_{i j}\left(x_{k l}\left(t-\tau_{k l}(t)\right)\right) x_{i j}(t) \\
& +\sum_{C^{k l} \in N_{r}(i, j)} C_{i j}^{k l}(t) \int_{-\infty}^{t} k_{i j}(t-s) g_{i j}\left(x_{k l}(s)\right) \mathrm{d} s x_{i j}(t)+I_{i j}(t),
\end{aligned}
$$


where $i=1,2, \ldots, n, j=1,2, \ldots, m . C_{i j}(t)$ denotes the cell at the $(i, j)$ position of the lattice at time $t$, the $r$-neighborhood $N_{r}(i, j)$ of $C_{i j}(t)$ is

$$
N_{r}(i, j)=\left\{C_{k l}(t): \max (|k-i|,|l-j|) \leq r, 1 \leq k \leq m, 1 \leq l \leq n\right\}
$$

$x_{i j}(t)$ is the activity of the cell $C_{i j}(t), I_{i j}(t)$ is the external input to $C_{i j}(t), a_{i j}\left(t, x_{i j}(t)\right)$ represents an appropriately behaved function of the cell $C_{i j}(t)$ at time $t ; B_{i j}^{k l}(t)$ and $C_{i j}^{k l}(t)$ are the connection or coupling strength of postsynaptic activity of the cell transmitted to the cell $C_{i j}(t)$ depending upon discrete delays and distributed delays, respectively; the activation functions $f_{i j}(\cdot)$ and $g_{i j}(\cdot)$ are continuous representing the output or firing rate of the cell $C_{k l}(t) ; \tau_{i j}(t)$ represent axonal signal transmission delays; $B_{i j}^{k l}(t), C_{i j}^{k l}(t), f_{i j}(\cdot), g_{i j}(\cdot), I_{i j}(t), \tau_{i j}(t)$ are all continuous almost periodic functions.

Since Bouzerdoum and Pinter described SICNNs as a new cellular neural networks [1-3], SICNNs have been extensively studied and found many important applications in different areas such as psychophysics, speech, perception, robotics, adaptive pattern recognition, vision, and image processing. There have been some results on the existence of periodic and almost solutions for SICNNs with discrete or distributed delays (distributed delay was first introduced in [4]) [5-21]. We find that all the behaved functions in the models in [5-21] are linear. Actually, $a_{i j}\left(t, x_{i j}(t)\right)$ may be nonlinear. Moreover, we find that the models in [5-20] are special cases of (1.1). For example, let $a_{i j}\left(t, x_{i j}(t)\right)=a_{i j}(t) x_{i j}(t)$, then $C_{i j}^{k l}(t) \equiv 0$ in [5], $B_{i j}^{k l}(t) \equiv 0$ in [7], $C_{i j}^{k l}(t) \equiv 0$ in [8], $a_{i j}(t), C_{i j}^{k l}(t)$ are constants and $B_{i j}^{k l}(t) \equiv 0$ in [9], $a_{i j}(t), B_{i j}^{k l}(t), \tau_{k l}(t)$ are constants, and $C_{i j}^{k l}(t) \equiv 0$ in $[10,11]$. To the best of our knowledge, few authors have considered the existence and global exponential stability of almost periodic solutions for SICNNs with nonlinear behaved functions, periodic coefficients and mixed delays. Obviously, (1.1) is general and is worth to continue to investigate its dynamical properties such as existence and global exponential stability of almost periodic solutions.

The main purpose of this paper is to get sufficient conditions on the existence and global exponential stability of almost periodic solutions for SICNNs (1.1) by using the Leray-Schauder fixed point theorem and differential inequality techniques. Our results are general and possess infinitely adjustable real parameters and can be deduced to many simple results, including some existing results as special cases. Therefore, our results provide a wider application criteria for neural networks.

The remaining part of this paper is organized as follows. We first state some useful definitions and lemmas in Section 2. In Section 3, we study the existence of almost periodic solutions of system (1.1) by using the Leray-Schauder's fixed point theorem. In Section 4, by using Lemma 2.7, we will derive sufficient conditions for the global exponential stability of the almost periodic solution of system (1.1). A useful corollary is also obtained. An illustrative example and its simulation are given in Section 5.

\section{Preliminaries}

For convenience, we denote

$$
x=\left\{x_{i j}(t)\right\}=\left(x_{11}(t), x_{12}(t), \ldots, x_{1 m}(t), \ldots, x_{n 1}(t), x_{n 2}(t), \ldots, x_{n m}(t)\right)^{T} .
$$


Definition 2.1. The continuous function $x_{i j}(t): \mathbb{R} \rightarrow \mathbb{R}$ is called almost periodic on $\mathbb{R}$, if for any $\varepsilon>0$, it is possible to find a real number $l=l(\varepsilon)>0$ such that, for any interval with length $l$, there exists a number $\tau=\tau(\varepsilon)$ in this interval such that $\left|x_{i j}(t+\tau)-x_{i j}(t)\right|<\varepsilon$, for any $t \in \mathbb{R}$.

Definition 2.2 (see [22, page 21]). Let $\mathbb{E}$ be a Banach space, $\mathbb{D}$ an open subset in $\mathbb{E}$ and $f(t, x) \in$ $C(\mathbb{R} \times \mathbb{D}, \mathbb{E})$. For $\forall x \in \mathbb{D}, f(t, x)$ is called uniformly almost periodic about $t$, if for any $\varepsilon>0$ and any compact subset $\mathbb{S} \subset \mathbb{D}$, there exists a real number $l=l(\varepsilon, \mathbb{S})>0$ such that, for any interval with length $l$, there exists a number $\tau=\tau(\varepsilon, \mathbb{S})$ in this interval such that $\|f(t+\tau, x)-f(t, x)\|<$ $\varepsilon$, for any $(t, x) \in \mathbb{R} \times \mathbb{S}$.

The initial condition $\varphi=\left\{\varphi_{i j}(s)\right\}$ of $(1.1)$ is of the form

$$
x_{i j}(s)=\varphi_{i j}(s), \quad s \in(-\infty, 0]
$$

where $\varphi_{i j}(s), i=1,2, \ldots, n, j=1, \ldots, m$, are continuous almost periodic solutions.

Definition 2.3. Let $x^{*}(t)$ be an almost periodic solution of (1.1) with initial value $\varphi^{*}$. If there exist constants $\alpha>0$ and $P>1$ such that for every solution $x(t)$ of (1.1) with initial value $\varphi$

$$
\left|x_{i j}(t)-x_{i j}^{*}(t)\right| \leq P\left\|\varphi-\varphi^{*}\right\| e^{-\alpha t}, \quad \forall t>0, i=1,2, \ldots, n, j=1,2, \ldots, m
$$

where $\left\|\varphi-\varphi^{*}\right\|=\max _{(i, j)} \sup _{s \leq 0}\left\{\left|\varphi_{i j}(s)-\varphi_{i j}^{*}(s)\right|\right\}$. Then $x^{*}(t)$ is said to be globally exponentially stable.

Lemma 2.4 (see [22, page 136]). Suppose that $f(t, x)$ is uniformly continuous on $\mathbb{R} \times \mathbb{S}$, where $\mathbb{S}$ is any compact set on $\mathbb{R}^{n}$, and that there exists a nonsingular matrix $P(t) \in C^{1}$, such that

(a) there exists a constant $\rho$ satisfies $\|P(t)\|<\rho$, for all $t \in \mathbb{R}$,

(b) the eigenvalues $\lambda_{i}(t), i=1,2, \ldots, n$, of $P(t)$ satisfy $\left|\lambda_{i}(t)\right| \geq \vartheta>0$, where $\vartheta$ is a constant, and there are $k$ negative eigenvalues, $n-k$ nonnegative eigenvalues,

(c) all the eigenvalues $\lambda_{i}(t, x), i=1,2, \ldots, n$, of the following symmetric matrix:

$$
M(t, x)=P(t) f(t, x)+f^{T}(t, x) P(t)+\dot{P}(t)
$$

satisfy

$$
\lambda_{i}(t, x) \leq-\delta<0, \quad \forall\|x\| \leq R_{0}
$$

where $\delta, R_{0}$ are constants.

Then, for any fixed $\phi(t) \in C\left(\mathbb{R}, \mathbb{R}^{n}\right)$ and any $t \in \mathbb{R}$ satisfying $\|\phi(t)\|<R_{0}$, the linear differential equation

$$
\dot{x}=f(t, \phi(t)) x
$$


admits an exponential dichotomy on $\mathbb{R}$ :

$$
\begin{gathered}
\left|X_{\phi}(t) Q_{\phi} X_{\phi}^{-1}(s)\right| \leq M \sqrt{\frac{\rho}{\vartheta}} e^{-(\delta / 2 \rho)(t-s)}, \quad t \geq s, \\
\left|X_{\phi}(t)\left(I-Q_{\phi}\right) X_{\phi}^{-1}(s)\right| \leq M \sqrt{\frac{\rho}{\vartheta}} e^{-(\delta / 2 \rho)(t-s)}, \quad s \geq t,
\end{gathered}
$$

where $X_{\phi}(t)$ is fundamental solution matrix of (2.6) satisfying $X_{\phi}(0)=I_{n \times n}, I_{n \times n}$ is the identity matrix, $Q_{\phi}$ is a constant projection, the constant $M$ has no relationship with $\phi(t)$.

Lemma 2.5 (see [22, page 139]). Suppose that the $n \times n$ matrix function $f(t, x)$ and the $n$ dimensional vector function $g(t, x)$ are uniformly almost periodic on $\mathbb{R} \times \mathbb{R}^{n}$, and that there exists real symmetric nonsingular matrix $P(t)$ satisfying the conditions (a) $-(c)$ in Lemma 2.4, then,

$$
\dot{x}=f(t, \phi) x+g(t, \phi)
$$

has a unique almost periodic solution $x(t)$, where $\phi \in C\left(\mathbb{R}, \mathbb{R}^{n}\right)$ is almost periodic function and

$$
x(t)=\int_{-\infty}^{t} X_{\phi}(s) Q_{\phi} X_{\phi}^{-1}(s) g(s, \phi(s)) d s-\int_{t}^{+\infty} X_{\phi}(s)\left(I-Q_{\phi}\right) X_{\phi}^{-1}(s) g(s, \phi(s)) d s .
$$

Lemma 2.6 (Leray-Schauder). Let $\mathbb{E}$ be a Banach space, and let the operator $\Phi: \mathbb{E} \rightarrow \mathbb{E}$ be completely continuous. If the set $\{\|x\| \mid x \in \mathbb{E}, x=\lambda \Phi x, 0<\lambda<1\}$ is bounded, then $\Phi$ has a fixed point in $\mathbb{T}$, where

$$
\mathbb{T}=\{x \mid x \in E,\|x\| \leq R\}, \quad R=\sup \{\|x\| \mid x=\lambda \Phi x, 0<\lambda<1\} .
$$

Lemma 2.7 (see [23]). Let $a \geq 0, b_{k} \geq 0(k=1,2, \ldots, m)$, the following inequality holds

$$
a \prod_{k=1}^{m} b_{k}^{q_{k}} \leq \frac{1}{r} \sum_{k=1}^{m} q_{k} b_{k}^{r}+\frac{1}{r} a^{r},
$$

where $q_{k}>0,(k=1,2, \ldots, m)$ is some constants, $\sum_{k=1}^{m} q_{k}=r-1$, and $r>1$.

Obviously, inequality (2.11) also holds for $a \geq 0, b_{k} \geq 0, r=1$, and $q_{k}=0, k=$ $1,2, \ldots, m$. Hence, we always assume that $a \geq 0, b_{k} \geq 0, r \geq 1$, and $q_{k} \geq 0, k=1,2, \ldots, m$ in (2.11) in the later sections of this paper.

Furthermore, throughout this paper, we assume that

$\left(\mathrm{H}_{1}\right) a_{i j}(t, u) \in C\left(\mathbb{R}^{2}, \mathbb{R}\right)$ is continuous almost periodic about the first argument and, there exists a positive continuous almost periodic function $\mu_{i j}(t)$ such that $\partial a_{i j}(t, u) / \partial u \geq \mu_{i j}(t), u \in \mathbb{R}$, and $a_{i j}(t, 0)=0, i=1,2, \ldots, n, j=1,2, \ldots, m$,

$\left(\mathrm{H}_{2}\right)$ there exist nonnegative constants $M_{i j}$ and $N_{i j}, i=1,2, \ldots, n, j=1,2, \ldots, m$, such that

$$
\left|f_{i j}(u)\right| \leq M_{i j}, \quad\left|g_{i j}(u)\right| \leq N_{i j}, \quad \forall u \in \mathbb{R}
$$


$\left(\mathrm{H}_{3}\right)$ the delay kernels $k_{i j}:[0,+\infty) \rightarrow \mathbb{R}$ are continuous, integrable and there are positive constants $\bar{k}_{i j}$ such that

$$
\int_{0}^{+\infty}\left|k_{i j}(s)\right| \mathrm{d} s \leq \bar{k}_{i j}, \quad i=1,2, \ldots, n, j=1,2, \ldots, m
$$

$\left(\mathrm{H}_{4}\right)$ there exists a constant $\alpha_{0}>0$ such that

$$
\int_{0}^{+\infty}\left|k_{i j}(s)\right| e^{\alpha_{0} s} \mathrm{~d} s<+\infty, \quad i=1,2, \ldots, n, j=1,2, \ldots, m
$$

$\left(\mathrm{H}_{5}\right)$ the following inequality holds:

$$
\max _{(i, j)} \sup _{t \in \mathbb{R}}\left\{\frac{\sum_{B^{k l} \in N_{r}(i, j)}\left|B_{i j}^{k l}(t)\right| M_{i j}+\sum_{C^{k l} \in N_{r}(i, j)}\left|C_{i j}^{k l}(t)\right| \bar{k}_{i j} N_{i j}}{\mu_{i j}(t)}\right\}=\eta<1 ;
$$

$\left(\mathrm{H}_{6}\right)$ there are nonnegative constants $\alpha_{i j}, \beta_{i j}$ such that

$$
\alpha_{i j}=\sup _{u \neq v}\left|\frac{f_{i j}(u)-f_{i j}(v)}{u-v}\right|, \quad \beta_{i j}=\sup _{u \neq v}\left|\frac{g_{i j}(u)-g_{i j}(v)}{u-v}\right|
$$

for all $u, v \in \mathbb{R}, u \neq v, i=1,2, \ldots, n, j=1,2, \ldots, m$.

\section{Existence of Almost Periodic Solutions}

Let $\xi_{i j}, i=1,2, \ldots, n, j=1,2, \ldots, m$ be constants. Make the following transformation:

$$
x_{i j}=\xi_{i j} y_{i j}(t), \quad i=1,2, \ldots, n, j=1,2, \ldots, m,
$$

then (1.1) can be reformulated as

$$
\begin{aligned}
y_{i j}^{\prime}(t)= & -\xi_{i j}^{-1} a_{i j}\left(t, \xi_{i j} y_{i j}(t)\right)+\sum_{B^{k l} \in N_{r}(i, j)} B_{i j}^{k l}(t) f_{i j}\left(\xi_{k l} y_{k l}\left(t-\tau_{k l}(t)\right)\right) y_{i j}(t) \\
& +\sum_{C^{k l} \in N_{r}(i, j)} C_{i j}^{k l}(t) \int_{-\infty}^{t} k_{i j}(t-s) g_{i j}\left(\xi_{k l} y_{k l}(s)\right) \mathrm{d} s y_{i j}(t)+\xi_{i j}^{-1} I_{i j}(t) .
\end{aligned}
$$


System (3.2) can be rewritten as

$$
\begin{aligned}
y_{i j}^{\prime}(t)= & -d_{i j}\left(t, y_{i j}(t)\right) y_{i j}(t)+\sum_{B^{k l} \in N_{r}(i, j)} B_{i j}^{k l}(t) f_{i j}\left(\xi_{k l} y_{k l}\left(t-\tau_{k l}(t)\right)\right) y_{i j}(t) \\
& +\sum_{C^{k l} \in N_{r}(i, j)} C_{i j}^{k l}(t) \int_{-\infty}^{t} k_{i j}(t-s) g_{i j}\left(\xi_{k l} y_{k l}(s)\right) \mathrm{d} s y_{i j}(t)+\xi_{i j}^{-1} I_{i j}(t),
\end{aligned}
$$

where $\left.d_{i j}\left(t, y_{i j}(t)\right) \triangleq\left(\partial a_{i j}(t, z) / \partial z\right)\right|_{z=e_{i j}}, e_{i j}$ is between 0 and $\xi_{i j} y_{i j}(t), e_{i j} \in \mathbb{R}$. By $\left(\mathrm{H}_{1}\right)$, we know that $a_{i j}\left(t, \xi_{i j} y_{i j}\right)$ is strictly monotone increasing about $y_{i j}$. Hence, $d_{i j}\left(t, y_{i j}(t)\right)$ is unique for any $y_{i j}(t)$. Obviously, $d_{i j}\left(t, y_{i j}(t)\right)$ is continuous almost periodic about the first argument and $d_{i j}\left(t, y_{i j}(t)\right) \geq \mu_{i j}(t)$.

Take $\mathbb{X}=\left\{\phi=\left\{\phi_{i j}(t)\right\} \mid \phi_{i j}: \mathbb{R} \rightarrow \mathbb{R}\right.$ is an almost periodic function, $i=1, \ldots, n$, $j=1, \ldots, m\}$. Then $\mathbb{X}$ is a Banach space with the norm

$$
\|\phi\|=\max _{(i, j)}\left\{\left|\phi_{i j}\right|_{0}\right\}, \quad\left|\phi_{i j}\right|_{0}=\sup _{t \in \mathbb{R}}\left|\phi_{i j}(t)\right|, \quad i=1, \ldots, n, j=1, \ldots, m .
$$

For for all $\phi \in \mathbb{X}$, we consider the following auxiliary equation:

$$
\begin{aligned}
y_{i j}^{\prime}(t)= & -d_{i j}\left(t, \phi_{i j}(t)\right) y_{i j}(t)+\sum_{B^{k l} \in N_{r}(i, j)} B_{i j}^{k l}(t) f_{i j}\left(\xi_{k l} \phi_{k l}\left(t-\tau_{k l}(t)\right)\right) \phi_{i j}(t) \\
& +\sum_{C^{k l} \in N_{r}(i, j)} C_{i j}^{k l}(t) \int_{-\infty}^{t} k_{i j}(t-s) g_{i j}\left(\xi_{k l} \phi_{k l}(s)\right) \mathrm{d} s \phi_{i j}(t)+\xi_{i j}^{-1} I_{i j}(t) .
\end{aligned}
$$

From $\left(\mathrm{H}_{1}\right)$, we know that

$$
d_{i j}\left(t, y_{i j}\right), \quad i=1,2, \ldots, n, j=1,2, \ldots, m,
$$

are uniformly almost periodic functions on $\mathbb{R} \times \mathbb{R}$. Since $\mu_{i j}(t), i=1,2, \ldots, n, j=1,2, \ldots, m$, are positive continuous almost periodic functions, there exists a positive constant $\delta$ such that

$$
d_{i j}\left(t, \phi_{i j}(t)\right) \geq \mu_{i j}(t) \geq \delta>0
$$

Hence, the conditions in Lemma 2.5 are satisfied (take $P(t)=I_{n m \times n m}$ ). 
According to the fact that $B_{i j}^{k l}(t), \phi_{i j}(t), C_{i j}^{k l}(t), I_{i j}(t)$ are almost periodic functions, in view of Lemma 2.5, we know that system (3.5) has a unique almost periodic solution

$$
\begin{aligned}
y^{\phi}(t)= & \left\{\int_{-\infty}^{t} e^{-\int_{s}^{t} d_{i j}\left(u, \phi_{i j}(u)\right) \mathrm{d} u}\right. \\
& \times\left[\sum_{B^{k l} \in N_{r}(i, j)} B_{i j}^{k l}(s) f_{i j}\left(\xi_{k l} \phi_{k l}\left(s-\tau_{k l}(s)\right)\right) \phi_{i j}(s)\right. \\
& \left.\left.+\sum_{C^{k l} \in N_{r}(i, j)} C_{i j}^{k l}(s) \int_{-\infty}^{s} k_{i j}(s-u) g_{i j}\left(\xi_{k l} \phi_{k l}(u)\right) \mathrm{d} u \phi_{i j}(s)+\xi_{i j}^{-1} I_{i j}(s)\right] \mathrm{d} s\right\} .
\end{aligned}
$$

Set a mapping $\Phi: \mathbb{X} \rightarrow \mathbb{X}$ by setting

$$
(\Phi \phi)(t)=y^{\phi}(t), \quad \forall \phi \in \mathbb{X}
$$

Before using Lemma 2.6 to obtain conditions of the existence of almost periodic solution for (1.1), we have to prove the following lemma.

Lemma 3.1. Suppose that $\left(H_{1}\right)-\left(H_{5}\right)$ hold. Then $\Phi: \mathbb{X} \rightarrow \mathbb{X}$ is completely continuous.

Proof. Under our assumptions, it is clear that the operator $\Phi$ is continuous. Next, we show that $\Phi$ is compact.

For any constant $D>0$, let $\Omega=\{\phi \mid \phi \in \mathbb{X},\|\phi\|<D\}$. Then, for any $\phi \in \Omega$, we have

$$
\begin{aligned}
\|(\Phi \phi)\|=\max _{(i, j)} \sup _{t \in \mathbb{R}}\left\{\mid \int_{-\infty}^{t} e^{-\int_{s}^{t} d_{i j}\left(u, \phi_{i j}(u)\right) \mathrm{d} u}\right. \\
\times \\
\times\left[\sum_{B^{k l} \in N_{r}(i, j)} B_{i j}^{k l}(s) f_{i j}\left(\xi_{k l} \phi_{k l}\left(s-\tau_{k l}(s)\right)\right) \phi_{i j}(s)\right. \\
\left.\left.\quad+\sum_{C^{k l} \in N_{r}(i, j)} C_{i j}^{k l}(s) \int_{-\infty}^{s} k_{i j}(s-u) g_{i j}\left(\xi_{k l} \phi_{k l}(u)\right) \mathrm{d} u \phi_{i j}(s)+\xi_{i j}^{-1} I_{i j}(s)\right] \mathrm{d} s \mid\right\}
\end{aligned}
$$




$$
\begin{gathered}
\leq \max _{(i, j)} \sup _{t \in \mathbb{R}}\left\{\int _ { - \infty } ^ { t } e ^ { - \int _ { s } ^ { t } \mu _ { i j } ( u ) \mathrm { d } u } \left[\left(\sum_{B^{k l} \in N_{r}(i, j)}\left|B_{i j}^{k l}(s)\right| M_{i j}+\sum_{C^{k l} \in N_{r}(i, j)}\left|C_{i j}^{k l}(s)\right| \bar{k}_{i j} N_{i j}\right)\right.\right. \\
\left.\left.\times\left|\phi_{i j}(s)\right|+\xi_{i j}^{-1}\left|I_{i j}(s)\right|\right] \mathrm{d} s\right\} \\
\leq \max _{(i, j)} \sup _{t \in \mathbb{R}}\left\{\int_{-\infty}^{t} e^{-\int_{s}^{t} \mu_{i j}(u) \mathrm{d} u} \mu_{i j}(s) \mathrm{d} s\right\} \eta\|\phi\|+\max _{(i, j)}\left\{\frac{\bar{I}_{i j}}{\xi_{i j} \underline{\mu}_{i j}}\right\}<\eta D+\max _{(i, j)}\left\{\frac{\bar{I}_{i j}}{\xi_{i j} \underline{\mu}_{i j}}\right\},
\end{gathered}
$$

where $\bar{I}_{i j}=\max _{t \in R}\left|I_{i j}(t)\right|, \underline{\mu}_{i j}=\inf _{t \in \mathbb{R}} \mu_{i j}(t)$. Hence, $\Phi(\Omega)$ is uniformly bounded.

By the definition of $\Phi$, we get

$$
\begin{aligned}
(\Phi \phi)_{i j}^{\prime}(t)=\frac{\mathrm{d}}{\mathrm{d} t}\left(\int_{-\infty}^{t} e^{-\int_{s}^{t} d_{i j}\left(u, \phi_{i j}(u)\right) \mathrm{d} u}\right. & \\
& \times\left[\sum_{B^{k l} \in N_{r}(i, j)} B_{i j}^{k l}(s) f_{i j}\left(\xi_{k l} \phi_{k l}\left(s-\tau_{k l}(s)\right)\right) \phi_{i j}(s)\right. \\
& \left.\left.+\sum_{C^{k l} \in N_{r}(i, j)} C_{i j}^{k l}(s) \int_{-\infty}^{s} k_{i j}(s-u) g_{i j}\left(\xi_{k l} \phi_{k l}(u)\right) \mathrm{d} u \phi_{i j}(s)+\xi_{i j}^{-1} I_{i j}(s)\right] \mathrm{d} s\right) \\
=-d_{i j}\left(t, \phi_{i j}(t)\right)\left(\Phi \phi_{i j}\right)(t)+\sum_{B^{k l} \in N_{r}(i, j)} B_{i j}^{k l}(t) f_{i j}\left(\xi_{k l} \phi_{k l}\left(t-\tau_{k l}(t)\right)\right) \phi_{i j}(t) & \\
& +\sum_{C^{k l} \in N_{r}(i, j)} C_{i j}^{k l}(t) \int_{-\infty}^{t} k_{i j}(t-s) g_{i j}\left(\xi_{k l} \phi_{k l}(s)\right) \mathrm{d} s \phi_{i j}(t)+\xi_{i j}^{-1} I_{i j}(t) .
\end{aligned}
$$

Since $d_{i j}\left(t, \phi_{i j}(t)\right), i=1,2, \ldots, n, j=1,2, \ldots, m$, are uniformly almost periodic functions on $\mathbb{R} \times \Omega$, there exists a positive constant $\Theta$ such that

$$
\left|d_{i j}\left(t, \phi_{i j}(t)\right)\right| \leq \Theta, \quad \text { for } t \in \mathbb{R}, \phi_{i j}(t) \in \Omega, i=1,2, \ldots, n, j=1,2, \ldots, m
$$


Hence,

$$
\begin{aligned}
\left\|(\Phi \phi)_{i j}^{\prime}(t)\right\| \leq & \Theta\left(\eta D+\max _{(i, j)}\left\{\frac{\bar{I}_{i j}}{\xi_{i j} \underline{\mu}_{i j}}\right\}\right) \\
& +\max _{(i, j)}\left\{\sum_{B^{k l} \in N_{r}(i, j)} \bar{B}_{i j}^{k l} M_{i j} D+\sum_{C^{k l} \in N_{r}(i, j)} \bar{C}_{i j}^{k l} \bar{k}_{i j} N_{i j} D+\xi_{i j}^{-1} \bar{I}_{i j}\right\},
\end{aligned}
$$

where $\bar{B}_{i j}^{k l}=\sup _{t \in R}\left|B_{i j}^{k l}(t)\right|, \bar{C}_{i j}^{k l}=\sup _{t \in \mathbb{R}}\left|C_{i j}^{k l}(t)\right|$. So, $\Phi(\Omega) \subseteq \mathbb{X}$ is a family of uniformly bounded and equicontinuous subsets. By using the Arzela-Ascoli theorem, $\Phi: \mathbb{X} \rightarrow \mathbb{X}$ is compact. Therefore, $\Phi: \mathbb{X} \rightarrow \mathbb{X}$ is completely continuous. This completes the proof.

Theorem 3.2. Suppose that $\left(H_{1}\right)-\left(H_{5}\right)$ hold. Let $\xi_{i j}, i=1,2, \ldots, n, j=1,2, \ldots, m$, be constants. Then system (1.1) has an almost periodic solution $x^{*}(t)$ with $\left\|x^{*}\right\| \leq \max _{(i, j)}\left\{\xi_{i j}\right\} \widetilde{R} \triangleq R_{0}$, where

$$
\tilde{R}=\frac{\max _{(i, j)}\left\{\bar{I}_{i j} / \xi_{i j} \underline{\mu}_{i j}\right\}}{1-\eta} .
$$

Proof. Let $\phi \in \mathbb{X}$. From Lemma 3.1, we get that $\Phi: \mathbb{X} \rightarrow \mathbb{X}$ is completely continuous. Consider the following operator equation:

$$
\phi=\lambda \Phi \phi, \quad \lambda \in(0,1)
$$

If $\phi$ is a solution of (3.15), we obtain

$$
\|\phi\| \leq\|\Phi \phi\| \leq \theta\|\phi\|+\max _{(i, j)}\left\{\frac{\bar{I}_{i j}}{\xi_{i j} \underline{\mu}_{i j}}\right\} .
$$

This and $\left(\mathrm{H}_{5}\right)$ imply that

$$
\|\phi\| \leq \widetilde{R}
$$

In view of Lemma 2.6, we obtain that $\Phi$ has a fixed point $\phi^{*}(t)$ with $\left\|\phi^{*}\right\| \leq \widetilde{R}$. From (3.5) and (3.8), we know that $\phi^{*}$ satisfies (3.3) and (3.2). Hence, system (3.2) has an almost periodic solution $\phi^{*}(t)=\left\{\phi_{i j}^{*}(t)\right\}$ with $\left\|\phi^{*}\right\| \leq \widetilde{R}$. It follows from (3.1) that $x^{*}(t)=\left\{x_{i j}^{*}(t)\right\}=\left\{\xi_{i j} \phi_{i j}^{*}(t)\right\}$ is one almost periodic solution of (1.1) with

$$
\left\|x^{*}\right\| \leq \max _{(i, j)}\left\{\xi_{i j}\right\} \widetilde{R} \triangleq R_{0} .
$$

This completes the proof. 


\section{Stability of Almost Periodic solution}

In this section, we prove that, under suitable conditions, the almost periodic solution obtained in Theorem 3.2 is globally exponentially stable.

Theorem 4.1. Assume that $\left(H_{1}\right)-\left(H_{4}\right)$ and $\left(H_{6}\right)$ hold and

$\left(\mathrm{H}_{7}\right)$ there are constants $o_{s}, \varepsilon_{s}, l_{s}, \gamma_{s}, \delta_{s}, d_{s} \in \mathbb{R}, q \geq 1, p_{s} \geq 0, \sigma>0, \xi_{i j}>0, s=1,2, \ldots, m^{\prime}+$ $1, i=1,2, \ldots, n, j=1,2, \ldots, m$, such that

$$
\begin{aligned}
\Lambda=\operatorname{maxsup}_{(i, j)}\{ & \left(-q \mu_{i j}(t)+q \sum_{B^{k l} \in N_{r}(i, j)}\left|B_{i j}^{k l}(t)\right| M_{i j}+q \sum_{C^{k l} \in N_{r}(i, j)}\left|C_{i j}^{k l}(t)\right| \bar{k}_{i j} N_{i j}\right. \\
& \left.+\sum_{B^{k l} \in N_{r}(i, j)} \sum_{s=1}^{m^{\prime}} p_{s}\left|B_{i j}^{k l}(t)\right|^{q o_{s}} \alpha_{i j}^{q \varepsilon_{s}} R_{0}^{q l_{s}}+\sum_{C^{k l} \in N_{r}(i, j)} \sum_{s=1}^{m^{\prime}} p_{s}\left|C_{i j}^{k l}(t)\right|^{q \gamma_{s}} \beta_{i j}^{q \delta_{s}} R_{0}^{q d_{s}}\right) \xi_{i j} \\
& +\sum_{B^{k l} \in N_{r}(i, j)}\left|B_{i j}^{k l}(t)\right|^{q o_{m^{\prime}+1}} \alpha_{i j}^{q \varepsilon_{m^{\prime}+1}} R_{0}^{q l_{m^{\prime}+1}} \xi_{k l} \\
& \left.+\sum_{C^{k l} \in N_{r}(i, j)}\left|C_{i j}^{k l}(t)\right|^{q \gamma_{m^{\prime}+1}} \beta_{i j}^{q \delta_{m^{\prime}+1}} R_{0}^{q d_{m^{\prime}+1}} \bar{k}_{i j}^{q} \xi_{k l}\right\} \leq-\sigma<0,
\end{aligned}
$$

where, $o_{m^{\prime}+1}+\sum_{s=1}^{m^{\prime}} o_{s} p_{s}=\varepsilon_{m^{\prime}+1}+\sum_{s=1}^{m^{\prime}} \varepsilon_{s} p_{s}=l_{m^{\prime}+1}+\sum_{s=1}^{m^{\prime}} l_{s} p_{s}=\gamma_{m^{\prime}+1}+\sum_{s=1}^{m^{\prime}} \gamma_{s} p_{s}=$ $\delta_{m^{\prime}+1}+\sum_{s=1}^{m^{\prime}} \delta_{s} p_{s}=d_{m^{\prime}+1}+\sum_{s=1}^{m^{\prime}} d_{s} p_{s}=1, \sum_{s=1}^{m^{\prime}} p_{s}=q-1$.

Then (1.1) has unique almost periodic solution, which is globally exponentially stable.

Proof. Obviously, that $\left(\mathrm{H}_{7}\right)$ holds implies that $\left(\mathrm{H}_{5}\right)$ holds. By Theorem 3.2, there exists an almost periodic solution $x^{*}(t)$ of (1.1) with initial value $\varphi^{*}(t)$ and $\left\|x^{*}\right\| \leq R_{0}$. Suppose that $x(t)$ is an arbitrary solution of (1.1) with initial value $\varphi(t)$. Set $z(t)=x(t)-x^{*}(t)$. Then, from (1.1), we have

$$
\begin{aligned}
& z_{i j}^{\prime}(t)=-\left[a_{i j}\left(t, x_{i j}(t)\right)-a_{i j}\left(t, x_{i j}^{*}(t)\right)\right] \\
&+\sum_{B^{k l} \in N_{r}(i, j)} B_{i j}^{k l}(t)\left(f_{i j}\left(x_{k l}\left(t-\tau_{k l}(t)\right)\right) x_{i j}(t)-f_{i j}\left(x_{k l}^{*}\left(t-\tau_{k l}(t)\right)\right) x_{i j}^{*}(t)\right) \\
&+\sum_{C^{k l} \in N_{r}(i, j)} C_{i j}^{k l}(t)\left(\int_{-\infty}^{t} k_{i j}(t-s) g_{i j}\left(x_{k l}(s)\right) \mathrm{d} s x_{i j}(t)-\int_{-\infty}^{t} k_{i j}(t-s) g_{i j}\left(x_{k l}^{*}(s)\right) \mathrm{d} s x_{i j}^{*}(t)\right), \\
& i=1,2, \ldots, n, j=1,2, \ldots, m .
\end{aligned}
$$


Set

$$
\begin{aligned}
& \Gamma_{i j}(\lambda, t)=\left(\lambda-q \mu_{i j}(t)+q \sum_{B^{k l} \in N_{r}(i, j)}\left|B_{i j}^{k l}(t)\right| M_{i j}+q \sum_{C^{k l} \in N_{r}(i, j)}\left|C_{i j}^{k l}(t)\right| \bar{k}_{i j} N_{i j}\right. \\
&\left.+\sum_{B^{k l} \in N_{r}(i, j)} \sum_{s=1}^{m^{\prime}} p_{s}\left|B_{i j}^{k l}(t)\right|^{q o_{s}} \alpha_{i j}^{q \varepsilon_{s}} R_{0}^{q l_{s}}+\sum_{C^{k l} \in N_{r}(i, j)} \sum_{s=1}^{m^{\prime}} p_{s}\left|C_{i j}^{k l}(t)\right|^{q \gamma_{s}} \beta_{i j}^{q \delta_{s}} R_{0}^{q d_{s}}\right) \xi_{i j} \\
&+\sum_{B^{k l} \in N_{r}(i, j)}\left|B_{i j}^{k l}(t)\right|^{q o_{m^{\prime}+1}} \alpha_{i j}^{q \varepsilon_{m^{\prime}+1}} R_{0}^{q l_{m^{\prime}+1}} e^{\lambda \tau_{k l}(t)} \xi_{k l} \\
&+\sum_{C^{k l} \in N_{r}(i, j)}\left|C_{i j}^{k l}(t)\right|^{q \gamma_{m^{\prime}+1}} \beta_{i j}^{q \delta_{m^{\prime}+1}} R_{0}^{q d_{m^{\prime}+1}}\left(\int_{0}^{+\infty}\left|k_{i j}(s)\right| e^{(\lambda / q) s} \mathrm{~d} s\right)^{q} \xi_{k l}, \\
& i=1,2, \ldots, n, \quad j=1,2, \ldots, m .
\end{aligned}
$$

Clearly, $\Gamma_{i j}(\lambda, t), i=1,2, \ldots, n, j=1,2, \ldots, m$, are continuous functions about $\lambda$ and almost periodic about $t$ on $\mathbb{R}$.

From $\left(\mathrm{H}_{7}\right)$, we get

$$
\Gamma_{i j}(0, t) \leq \Lambda \leq-\sigma<0, \quad i=1,2, \ldots, n, j=1,2, \ldots, m .
$$

In addition to

$$
\begin{aligned}
\frac{\partial \Gamma_{i j}(\lambda, t)}{\partial \lambda}= & \xi_{i j}+\sum_{B^{k l} \in N_{r}(i, j)}\left|B_{i j}^{k l}(t)\right|^{q o_{m^{\prime}+1}} \alpha_{i j}^{q \varepsilon_{m^{\prime}+1}} R_{0}^{q l_{m^{\prime}+1}} \tau_{k l}(t) e^{\lambda \tau_{k l}(t)} \xi_{k l} \\
& +\sum_{C^{k l} \in N_{r}(i, j)}\left|C_{i j}^{k l}(t)\right|^{q \gamma_{m^{\prime}+1}} \beta_{i j}^{q \delta_{m^{\prime}+1}} R_{0}^{q d_{m^{\prime}+1}}\left(\int_{0}^{+\infty}\left|k_{i j}(s)\right| s e^{(\lambda / q) s} \mathrm{~d} s\right)^{q} \xi_{k l}>0
\end{aligned}
$$

and $\Gamma_{i j}(+\infty, t)=+\infty$ for $\forall t \in R$, we obtain that $\Gamma_{i j}(\lambda, t), i=1,2, \ldots, n, j=1,2, \ldots, m$, are strictly monotone increasing functions about $\lambda$. Therefore, for any $i \in\{1,2, \ldots, n\}$, $j \in\{1,2, \ldots, m\}$, there is unique real number $\lambda_{0}^{i j}$ such that

$$
\sup _{t \in R} \Gamma_{i j}\left(\lambda_{0}^{i j}, t\right)=0
$$

In view of (4.4), we have $\lambda_{0}^{i j}>0$. Let $\theta=\min \left\{\lambda_{0}^{11}, \lambda_{0}^{12}, \ldots, \lambda_{0}^{n m}\right\}$, we obtain

$$
\sup _{t \in R} \Gamma_{i j}(\theta, t) \leq 0, \quad i=1,2, \ldots, n, j=1,2, \ldots, m .
$$


Now, we choose a positive constant $d$ such that $q d \xi_{i j} \geq 1, i=1,2, \ldots, n, j=1,2, \ldots, m$. It is obvious that

$$
\left|z_{i}(t)\right|^{q} \leq\left\|\varphi-\varphi^{*}\right\|^{q} \leq q d \xi_{i}\left\|\varphi-\varphi^{*}\right\|^{q} e^{-\theta t}, \quad \text { for } \in(-\infty, 0], i=1,2, \ldots, n,
$$

where $\left\|\varphi-\varphi^{*}\right\|$ is defined as that in Definition 2.3.

Let $V_{i j}(t)=(1 / q) e^{\theta t}\left|z_{i j}(t)\right|^{q}, i=1,2, \ldots, n, j=1,2, \ldots, m$. In view of (4.2) and (2.11), for $t \geq 0$, we obtain

$$
\begin{aligned}
& \frac{\mathrm{d}^{+} V_{i j}(t)}{\mathrm{d} t} \\
& =e^{\theta t}\left|z_{i j}(t)\right|^{q-1} \\
& \times \operatorname{sgn} z_{i j}\left\{-\left[a_{i j}\left(t, x_{i j}(t)\right)-a_{i j}\left(t, x_{i j}^{*}(t)\right)\right]\right. \\
& +\sum_{B^{k l} \in N_{r}(i, j)} B_{i j}^{k l}(t)\left(f_{i j}\left(x_{k l}\left(t-\tau_{k l}(t)\right)\right) x_{i j}(t)-f_{i j}\left(x_{k l}^{*}\left(t-\tau_{k l}(t)\right)\right) x_{i j}^{*}(t)\right) \\
& +\sum_{C^{k l} \in N_{r}(i, j)} C_{i j}^{k l}(t)\left(\int_{-\infty}^{t} k_{i j}(t-s) g_{i j}\left(x_{k l}(s)\right) \mathrm{d} s x_{i j}(t)\right. \\
& \left.\left.-\int_{-\infty}^{t} k_{i j}(t-s) g_{i j}\left(x_{k l}^{*}(s)\right) \mathrm{d} s x_{i j}^{*}(t)\right)\right\}+\frac{1}{q} \theta e^{\theta t}\left|z_{i j}(t)\right|^{q} \\
& \leq e^{\theta t}\left|z_{i j}(t)\right|^{q-1} \\
& \times\left\{-\mu_{i j}(t)\left|z_{i j}(t)\right|+\sum_{B^{k l} \in N_{r}(i, j)}\left|B_{i j}^{k l}(t)\right|\right. \\
& \times\left(\left|f_{i j}\left(x_{k l}\left(t-\tau_{k l}(t)\right)\right)\right|\left|z_{i j}(t)\right|+\left|f_{i j}\left(x_{k l}\left(t-\tau_{k l}(t)\right)\right)-f_{i j}\left(x_{k l}^{*}\left(t-\tau_{k l}(t)\right)\right)\right|\left|x_{i j}^{*}(t)\right|\right) \\
& +\sum_{C^{k l} \in N_{r}(i, j)}\left|C_{i j}^{k l}(t)\right|\left(\int_{-\infty}^{t}\left|k_{i j}(t-s)\right|\left|g_{i j}\left(x_{k l}(s)\right)\right| \mathrm{d} s\left|z_{i j}(t)\right|\right. \\
& \left.\left.+\int_{-\infty}^{t}\left|k_{i j}(t-s)\right|\left|g_{i j}\left(x_{k l}(s)\right)-g_{i j}\left(x_{k l}^{*}(s)\right)\right| \mathrm{d} s\left|x_{i j}^{*}(t)\right|\right)\right\}+\frac{1}{q} \theta e^{\theta t}\left|z_{i j}(t)\right|^{q}
\end{aligned}
$$




$$
\begin{aligned}
& \leq e^{\theta t}\left|z_{i j}(t)\right|^{q-1} \\
& \times\left\{-\mu_{i j}(t)\left|z_{i j}(t)\right|+\sum_{B^{k l} \in N_{r}(i, j)}\left|B_{i j}^{k l}(t)\right|\left(M_{i j}\left|z_{i j}(t)\right|+\alpha_{i j} R_{0}\left|z_{k l}\left(t-\tau_{k l}(t)\right)\right|\right)\right. \\
& \left.+\sum_{C^{k l} \in N_{r}(i, j)}\left|C_{i j}^{k l}(t)\right|\left(\bar{k}_{i j} N_{i j}\left|z_{i j}(t)\right|+\beta_{i j} R_{0} \int_{-\infty}^{t}\left|k_{i j}(t-s)\right|\left|z_{k l}(s)\right| \mathrm{d} s\right)\right\} \\
& +\frac{1}{q} \theta e^{\theta t}\left|z_{i j}(t)\right|^{q} \\
& \leq \\
& +\theta-q \mu_{i j}(t)+q \sum_{B^{k l} \in N_{r}(i, j)}\left|B_{i j}^{k l}(t)\right| M_{i j}+q \sum_{C^{k l} \in N_{r}(i, j)}\left|C_{i j}^{k l}(t)\right| \bar{k}_{i j} N_{i j} \\
& \left.+\sum_{B^{k l} \in N_{r}(i, j)} \sum_{s=1}^{m^{\prime}} p_{s}\left|B_{i j}^{k l}(t)\right|^{q \rho_{s}} \alpha_{i j}^{q \varepsilon_{s}} R_{0}^{q l_{s}}+\sum_{C^{k l} \in N_{r}(i, j)} \sum_{s=1}^{m^{\prime}} p_{s}\left|C_{i j}^{k l}(t)\right|^{q \gamma_{s}} \beta_{i j}^{q \delta_{s}} R_{0}^{q d_{s}}\right) V_{i j}(t) \\
& +\sum_{B^{k l} \in N_{r}(i, j)}\left|B_{i j}^{k l}(t)\right|^{q O_{m^{\prime}+1}} \alpha_{i j}^{q \varepsilon_{m^{\prime}+1}} R_{0}^{q l_{m^{\prime}+1}} e^{\theta \tau_{k l}(t)} V_{k l}\left(t-\tau_{k l}(t)\right) \\
& +\left.C_{i j}^{k l}(t)\right|^{q \gamma_{m^{\prime}+1}} \beta_{i j}^{q \delta_{m^{\prime}+1}} R_{0}^{q d_{m^{\prime}+1}}\left(\int_{-\infty}^{t}\left|k_{i j}(t-s)\right| e^{(\theta / q)(t-s)} V_{k l}(s)^{1 / q} \mathrm{~d} s\right)^{q} .
\end{aligned}
$$

We now prove that the following statement is true:

$$
V_{i j}(t)=\frac{1}{q} e^{\theta t}\left|z_{i j}(t)\right|^{q} \leq d \xi_{i j}\left\|\phi-\phi^{*}\right\|^{q}, \quad i=1,2, \ldots, n, j=1,2, \ldots, m, \forall t>0 .
$$

Contrarily, there must exist some $i \in\{1,2, \ldots, n\}, j \in\{1,2, \ldots, m\}$, and $\tilde{t}>0$, such that

$$
V_{i j}(\tilde{t})=d \xi_{i j}\left\|\phi-\phi^{*}\right\|^{q}, \quad \frac{\mathrm{d}^{+} V_{i j}(\tilde{t})}{\mathrm{d} t}>0, \quad V_{k l}(t) \leq d \xi_{k l}\left\|\phi-\phi^{*}\right\|^{q},
$$


for all $t \in(-\infty, \tilde{t}], k=1,2, \ldots, n, l=1,2, \ldots, m$. Together with (4.9), (4.11), and (4.7), we obtain

$$
\begin{aligned}
0<\frac{\mathrm{d}^{+} V_{i j}(\tilde{t})}{\mathrm{d} t} & \\
\leq d\left\|\phi-\phi^{*}\right\|^{q}\{( & \theta-q \mu_{i j}(\tilde{t})+q \sum_{B^{k l} \in N_{r}(i, j)}\left|B_{i j}^{k l}(\tilde{t})\right| M_{i j}+q \sum_{C^{k l} \in N_{r}(i, j)}\left|C_{i j}^{k l}(\tilde{t})\right| \bar{k}_{i j} N_{i j} \\
& \left.+\sum_{B^{k l} \in N_{r}(i, j)} \sum_{s=1}^{m^{\prime}} p_{s}\left|B_{i j}^{k l}(\tilde{t})\right|^{q o_{s}} \alpha_{i j}^{q \varepsilon_{s}} R_{0}^{q l_{s}}+\sum_{C^{k l} \in N_{r}(i, j)} \sum_{s=1}^{m^{\prime}} p_{s}\left|C_{i j}^{k l}(\tilde{t})\right|^{q \gamma_{s}} \beta_{i j}^{q \delta_{s}} R_{0}^{q d_{s}}\right) \xi_{i j} \\
& +\sum_{B^{k l} \in N_{r}(i, j)}\left|B_{i j}^{k l}(\tilde{t})\right|^{q o_{m^{\prime}+1}} \alpha_{i j}^{q \varepsilon_{m^{\prime}+1}} R_{0}^{q l_{m^{\prime}+1}} e^{\theta \tau_{k l}(\tilde{t})} \xi_{k l} \\
& \left.+\sum_{C^{k l} \in N_{r}(i, j)}\left|C_{i j}^{k l}(\tilde{t})\right|^{q \gamma_{m^{\prime}+1}} \beta_{i j}^{q \delta_{m^{\prime}+1}} R_{0}^{q d_{m^{\prime}+1}}\left(\int_{0}^{+\infty}\left|k_{i j}(s)\right| e^{(\theta / q) s} \mathrm{~d} s\right)^{q} \xi_{k l}\right\} \leq 0,
\end{aligned}
$$

which is a contradiction. Hence, (4.10) holds. It follows that

$$
\left|x_{i j}(t)-x_{i j}^{*}(t)\right|=\left|z_{i j}(t)\right| \leq\left(q d \xi_{i j}\right)^{1 / q}\left\|\varphi-\varphi^{*}\right\| e^{-(\theta / q) t}, \quad \forall t>0, i=1,2, \ldots, n, j=1,2, \ldots, m .
$$

Let $P=\max _{(i, j)}\left\{\left(q d \xi_{i j}\right)^{1 / q}+1\right\}$, then

$$
\left|x_{i j}(t)-x_{i j}^{*}(t)\right| \leq P\left\|\varphi-\varphi^{*}\right\| e^{-(\theta / q) t}, \quad \forall t>0, i=1,2, \ldots, n, j=1,2, \ldots, m .
$$

By means of Definition 2.3, the almost periodic solution $x^{*}(t)$ of $(1.1)$ is globally exponentially stable. This completes the proof.

Since Theorem 3.2 is general, the expression of $\left(\mathrm{H}_{7}\right)$ is complex. In order to check the applicability of the results easily, we give the following corollary.

Corollary 4.2. Assume that $\left(H_{1}\right)-\left(H_{4}\right)$ and $\left(H_{6}\right)$ hold. Furthermore, if one of the following conditions holds 
$\left(\mathrm{A}_{1}\right)$ there are constants $\sigma>0, q \geq 1, \xi_{i j}>0, i=1,2, \ldots, n, j=1,2, \ldots, m$, such that

$$
\begin{aligned}
\max _{(i, j)} \sup _{t \in R}\{ & \left(-q \mu_{i j}(t)+q \sum_{B^{k l} \in N_{r}(i, j)}\left|B_{i j}^{k l}(t)\right| M_{i j}+q \sum_{C^{k l} \in N_{r}(i, j)}\left|C_{i j}^{k l}(t)\right| \bar{k}_{i j} N_{i j}\right. \\
& \left.+(q-1) \sum_{B^{k l} \in N_{r}(i, j)}\left|B_{i j}^{k l}(t)\right|+(q-1) \sum_{C^{k l} \in N_{r}(i, j)}\left|C_{i j}^{k l}(t)\right|\right) \xi_{i j} \\
& \left.+\sum_{B^{k l} \in N_{r}(i, j)}\left|B_{i j}^{k l}(t)\right| \alpha_{i j}^{q} R_{0}^{q} \xi_{k l}+\sum_{C^{k l} \in N_{r}(i, j)}\left|C_{i j}^{k l}(t)\right| \beta_{i j}^{q} R_{0}^{q} \bar{k}_{i j}^{q} \xi_{k l}\right\} \leq-\sigma<0,
\end{aligned}
$$

$\left(\mathrm{A}_{2}\right)$ there are constants $\sigma>0, \xi_{i j}>0, i=1,2, \ldots, n, j=1,2, \ldots, m$, such that

$$
\begin{aligned}
\max _{(i, j)} \sup _{t \in R}\{ & \left(-2 \mu_{i j}(t)+2 \sum_{B^{k l} \in N_{r}(i, j)}\left|B_{i j}^{k l}(t)\right| M_{i j}+2 \sum_{C^{k l} \in N_{r}(i, j)}\left|C_{i j}^{k l}(t)\right| \bar{k}_{i j} N_{i j}\right. \\
& \left.+\sum_{B^{k l} \in N_{r}(i, j)}\left|B_{i j}^{k l}(t)\right|+\sum_{C^{k l} \in N_{r}(i, j)}\left|C_{i j}^{k l}(t)\right|\right) \xi_{i j} \\
& \left.+\sum_{B^{k l} \in N_{r}(i, j)}\left|B_{i j}^{k l}(t)\right| \alpha_{i j}^{2} R_{0}^{2} \xi_{k l}+\sum_{C^{k l} \in N_{r}(i, j)}\left|C_{i j}^{k l}(t)\right| \beta_{i j}^{2} R_{0}^{2} \bar{k}_{i j}^{2} \xi_{k l}\right\} \leq-\sigma<0,
\end{aligned}
$$

$\left(\mathrm{A}_{3}\right)$ there are constants $\sigma>0, \xi_{i j}>0, i=1,2, \ldots, n, j=1,2, \ldots, m$, such that

$$
\begin{aligned}
\max _{(i, j)} \sup _{t \in R}\{ & \left(-\mu_{i j}(t)+\sum_{B^{k l} \in N_{r}(i, j)}\left|B_{i j}^{k l}(t)\right| M_{i j}+\sum_{C^{k l} \in N_{r}(i, j)}\left|C_{i j}^{k l}(t)\right| \bar{k}_{i j} N_{i j}\right) \xi_{i j} \\
& \left.+\sum_{B^{k l} \in N_{r}(i, j)}\left|B_{i j}^{k l}(t)\right| \alpha_{i j} R_{0} \xi_{k l}+\sum_{C^{k l} \in N_{r}(i, j)}\left|C_{i j}^{k l}(t)\right| \beta_{i j} R_{0} \bar{k}_{i j} \xi_{k l}\right\} \leq-\sigma<0 .
\end{aligned}
$$
stable.

Then (1.1) has exactly one almost periodic solution, which is globally exponentially

Proof. Let $m^{\prime}=1, o_{1}=o_{2}=\gamma_{1}=\gamma_{2}=1 / q, \varepsilon_{1}=l_{1}=\delta_{1}=d_{1}=0$, and $\varepsilon_{2}=l_{2}=\delta_{2}=d_{2}=1$, $i=1,2, \ldots, n, j=1,2, \ldots, m$, in $\left(\mathrm{H}_{7}\right)$, respectively, then $\left(\mathrm{H}_{7}\right)$ turns to $\left(\mathrm{A}_{1}\right)$. proof.

Let $q=2$ and $q=1$ in $\left(\mathrm{A}_{1}\right)$, respectively, then we get $\left(\mathrm{A}_{2}\right)$ and $\left(\mathrm{A}_{3}\right)$. This completes the

Remark 4.3. Corollary 4.2 can still be simplified into many simple results, which include the results in [7-11]. For example, authors of [9] considered a special case of our model (1.1) and obtained sufficient conditions of existence and exponential stability of almost periodic 
solutions for their model. Although the results of [9] and ours are completely different, we claim that the results in [9] are also special cases of the results in this paper. The model considered in [9] is

$$
x_{i j}^{\prime}(t)=-a_{i j} x_{i j}(t)+\sum_{C^{k l} \in N_{r}(i, j)} C_{i j}^{k l} \int_{0}^{\infty} k_{i j}(s) g\left(x_{k l}(t-s)\right) \mathrm{d} s x_{i j}(t)+I_{i j}(t),
$$

where $a_{i j}, C_{i j}^{k l} \geq 0,|g(x)| \leq M$, and $|g(u)-g(\mathrm{v})| \leq \mu|u-v|$ for $u, v \in R$. The other arguments are same as this paper. Obviously, system (4.18) is a special case of system (1.1). The main theorem in [9] is the following.

Corollary 4.4. Assume that there exist nonnegative constants $I, q$, and $\eta$ such that $\max _{(i, j)}\left\{\sum_{C^{k l} \in N_{r}(i, j)} C_{i j}^{k l} \bar{k}_{i j} M / a_{i j}\right\}=\eta<1, I=\max _{(i, j)}\left\{I_{i j} / a_{i j}\right\}, q=\eta(1+\mu I /(1-\eta) M)<1$. Then system (4.18) has exactly one almost periodic solution, which is globally exponentially stable.

Now we prove that Corollary 4.4 is a special case of Corollary 4.2. Comparing (1.1) with (4.18), we have $\mu_{i j}(t)=a_{i j},\left|C_{i j}^{k l}(t)\right|=C_{i j}^{k l},\left|B_{i j}^{k l}(t)\right|=0, N_{i j}=M$. Let $\xi_{i j}=1, i=$ $1,2 \ldots, n, j=1,2 \ldots, m$. According to the proof of Theorem 3.2, we have $R_{0}=I /(1-\eta)$. So, $q=$ $\eta\left(1+\mu R_{0} / M\right)<1 \Leftrightarrow \eta\left(\left(M+\mu R_{0}\right) / M\right)<1$. In view of the condition $\sum_{C^{k l} \in N_{r}(i, j)} C_{i j}^{k l} \bar{k}_{i j} M / a_{i j} \leq$ $\eta$, we have

$$
\frac{\sum_{C^{k l} \in N_{r}(i, j)} C_{i j}^{k l} \bar{k}_{i j}}{a_{i j}}\left(M+\mu R_{0}\right)<1,
$$

which is equivalent to

$$
-a_{i j}+M \sum_{C^{k l} \in N_{r}(i, j)} C_{i j}^{k l} \bar{k}_{i j}+\mu R_{0} \sum_{C^{k l} \in N_{r}(i, j)} C_{i j}^{k l} \bar{k}_{i j}<0 .
$$

The expression is a special case of $\left(\mathrm{A}_{3}\right)$ in Corollary 4.2.

Similarly, from Corollary 4.2 , we can get the main results of $[5,11]$ by the condition $\left(\mathrm{A}_{3}\right)$ (restrict in the case of periodic system in [5]) and the main results of [10] by condition $\left(A_{2}\right)$. Hence, the results of this paper are useful and important.

Remark 4.5. In (1.1), if $f_{i j}(u), g_{i j}(u), u \in \mathbb{R}$ are replaced by $f_{i j}(t, u), g_{i j}(t, u), t, u \in \mathbb{R}$, where $f_{i j}(t, u), g_{i j}(t, u)$ are bounded and almost periodic functions about the first argument, then, by the same methods used in this paper, we can also get some general results similar to Theorem 4.1 and Corollary 4.2 and extend and improve the main results in [12].

Remark 4.6. Note that condition $\left(\mathrm{H}_{7}\right)$ in Theorem 4.1 possesses infinitely adjustable real parameters. This enables neural networks workers to obtain many special conditions on the existence and global exponential stability of almost periodic solutions and periodic solutions in the designs and applications of neural networks. We can offer many different corollaries by changing the parameters in $\left(\mathrm{H}_{7}\right)$, not only Corollary 4.2. Hence, our results provide many criteria for neural network's application. 


\section{A Numerical Example}

Consider the following SICNNs with mixed delays:

$$
\begin{aligned}
x_{i j}^{\prime}(\mathrm{t})= & -a_{i j}\left(t, x_{i j}(t)\right)+\sum_{B^{k l} \in N_{r}(i, j)} B_{i j}^{k l}(t) f_{i j}\left(x_{k l}\left(t-\tau_{k l}(t)\right)\right) x_{i j}(t) \\
& +\sum_{C^{k l} \in N_{r}(i, j)} C_{i j}^{k l}(t) \int_{-\infty}^{t} k_{i j}(t-s) g_{i j}\left(x_{k l}(s)\right) \mathrm{d} s x_{i j}(t)+I_{i j}(t),
\end{aligned}
$$

where $r=1, \tau_{i j}(t)=(\cos t)^{2}, i, j=1,2,3$. Take $a_{11}(t)=6 x-\cos x+x \sin t, a_{12}(t)=6 x+$ $0.5 \sin x+x \cos t, a_{13}(t)=5 x+\cos x+x \sin t, a_{21}(t)=7 x+\sin x+x \sin t, a_{22}(t)=7 x-$ $\cos x-x \sin t, a_{23}(t)=5 x-\cos x+x \cos t, a_{31}(t)=6 x+\sin x+x \cos t, a_{32}(t)=6 x+\cos x+$ $x \sin t, a_{33}(t)=6 x-\sin x+x \cos t, f_{i j}(x)=g_{i j}(x)=0.1 \sin x, k_{i j}(t)=e^{-t} \sin t$, and

$$
\begin{gathered}
{\left[\begin{array}{lll}
B_{11}(t) & B_{12}(t) & B_{13}(t) \\
B_{21}(t) & B_{22}(t) & B_{23}(t) \\
B_{31}(t) & B_{32}(t) & B_{33}(t)
\end{array}\right]=\left[\begin{array}{ccc}
0.1 \cos t & 0.2 \sin t & 0 \\
0.3 \cos t & 0 & 0.3 \sin t \\
0 & 0.4 \cos t & 0.2 \sin t
\end{array}\right],} \\
{\left[\begin{array}{lll}
C_{11}(t) & C_{12}(t) & C_{13}(t) \\
C_{21}(t) & C_{22}(t) & C_{23}(t) \\
C_{31}(t) & C_{32}(t) & C_{33}(t)
\end{array}\right]=\left[\begin{array}{ccc}
0.2 \cos t & 0.4 \sin t & 0.3 \cos t \\
0.6 \cos t & 0 & 0.5 \sin t \\
0.5 \sin t & 0.6 \cos t & 0.5 \sin t
\end{array}\right],} \\
{\left[\begin{array}{lll}
I_{11}(t) & I_{12}(t) & I_{13}(t) \\
I_{21}(t) & I_{22}(t) & I_{23}(t) \\
I_{31}(t) & I_{32}(t) & I_{33}(t)
\end{array}\right]=\left[\begin{array}{ccc}
2 \cos \sqrt{2} t & \sin \sqrt{3} t & 1+2 \cos \sqrt{2} t \\
1+\cos \sqrt{2} t & 2 \cos \sqrt{2} t & 1-\sin \sqrt{3} t \\
2 \sin \sqrt{2} t & 1-\cos \sqrt{3} t & 3 \sin \sqrt{2} t
\end{array}\right] .}
\end{gathered}
$$

Then $\mu_{11}(t)=5+\sin t, \mu_{12}(t)=5.5+\cos t, \mu_{13}(t)=4+\sin t, \mu_{21}(t)=6+\sin t, \mu_{22}(t)=$ $6-\sin t, \mu_{23}(t)=4+\cos t, \mu_{31}(t)=5+\cos t, \mu_{32}(t)=5+\sin t, \mu_{33}(t)=5+\cos t, \alpha_{i j}(t)=\beta_{i j}(t)=$ $M_{i j}=N_{i j}=0.1$.

$$
\begin{array}{rr}
\sum_{B^{k l} \in N_{r}(1,1)}\left|B_{11}^{k l}(t)\right|=0.4|\cos t|+0.2|\sin t|, & \sum_{B^{k l} \in N_{r}(1,2)}\left|B_{12}^{k l}(t)\right|=0.4|\cos t|+0.5|\sin t|, \\
\sum_{B^{k l} \in N_{r}(1,3)}\left|B_{13}^{k l}(t)\right|=0.5|\sin t|, & \sum_{B^{k l} \in N_{r}(2,1)}\left|B_{21}^{k l}(t)\right|=0.4|\cos t|+0.6|\sin t|,
\end{array}
$$




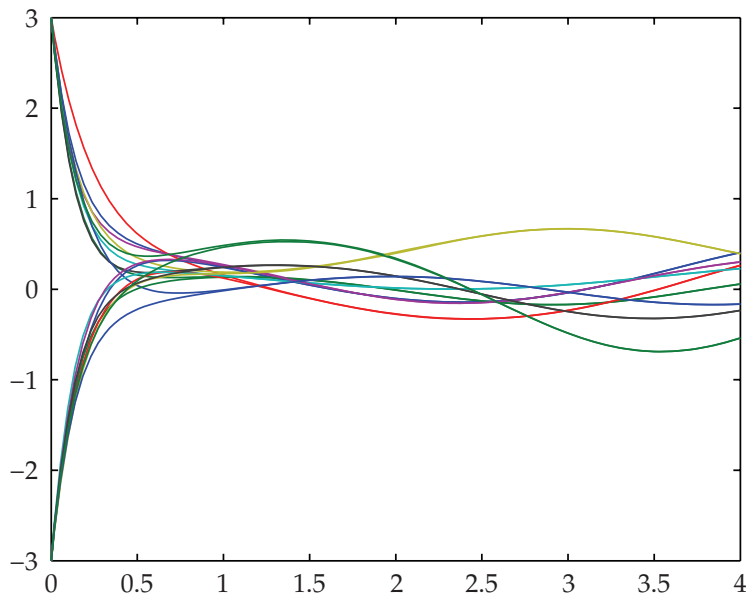

Figure 1: States of networks (5.1) with $t \in[0,4]$.

$$
\begin{array}{rlrl}
\sum_{B^{k l} \in N_{r}(2,2)}\left|B_{22}^{k l}(t)\right|=0.8|\cos t|+0.7|\sin t|, & & \sum_{B^{k l} \in N_{r}(2,3)}\left|B_{23}^{k l}(t)\right|=0.7|\cos t|+0.4|\sin t|, \\
\sum_{B^{k l} \in N_{r}(3,1)}\left|B_{31}^{k l}(t)\right|=0.3|\cos t|+0.4|\sin t|, & & \sum_{B^{k l} \in N_{r}(3,2)}\left|B_{32}^{k l}(t)\right|=0.7|\cos t|+0.5|\sin t|, \\
\sum_{B^{k l} \in N_{r}(3,3)}\left|B_{33}^{k l}(t)\right|=0.4|\cos t|+0.5|\sin t|, & \sum_{C^{k l} \in N_{r}(1,1)}\left|C_{11}^{k l}(t)\right|=0.8|\cos t|+0.4|\sin t|, \\
\sum_{C^{k l} \in N_{r}(1,2)}\left|C_{12}^{k l}(t)\right|=1.1|\cos t|+0.9|\sin t|, & \sum_{C^{k l} \in N_{r}(1,3)}\left|C_{13}^{k l}(t)\right|=0.3|\cos t|+0.9|\sin t|, \\
\sum_{C^{k l} \in N_{r}(2,1)}\left|C_{21}^{k l}(t)\right|=1.4|\cos t|+0.9|\sin t|, & \sum_{C^{k l} \in N_{r}(2,3)}\left|C_{22}^{k l}(t)\right|=1.7|\cos t|+1.9|\sin t|, \\
\sum_{C^{k l} \in N_{r}(3,2)}\left|C_{23}^{k l}(t)\right|=0.9|\cos t|+1.4|\sin t|, & & \sum_{C^{k l} \in N_{r}(3,1)}\left|C_{31}^{k l}(t)\right|=1.2|\cos t|+0.5|\sin t|,
\end{array}
$$

Take $\xi_{i j}=1, i, j=1,2,3$. Computing by Matlab toolbox, we have $\eta \approx 0.0721<1$, $R_{0}=\bar{I}_{13} / \underline{a}_{13}(1-\eta) \approx 1.0777$, and $\Lambda \approx-2.6849<0$. It is easy to check that all the conditions needed in Corollary 4.2 with $\left(\mathrm{A}_{3}\right)$ are satisfied. Therefore, system (5.1) has a unique almost periodic solution $x^{*}(t)$ with $\left\|x^{*}\right\| \leq 1.0777$, which is globally exponentially stable. See Figures 1 and 2.

Remark 5.1. In (5.1), the behaved functions are all nonlinear. So, all the results in [5-20] and the references therein are inapplicable for (5.1). This means that the results of this paper are new and useful. 


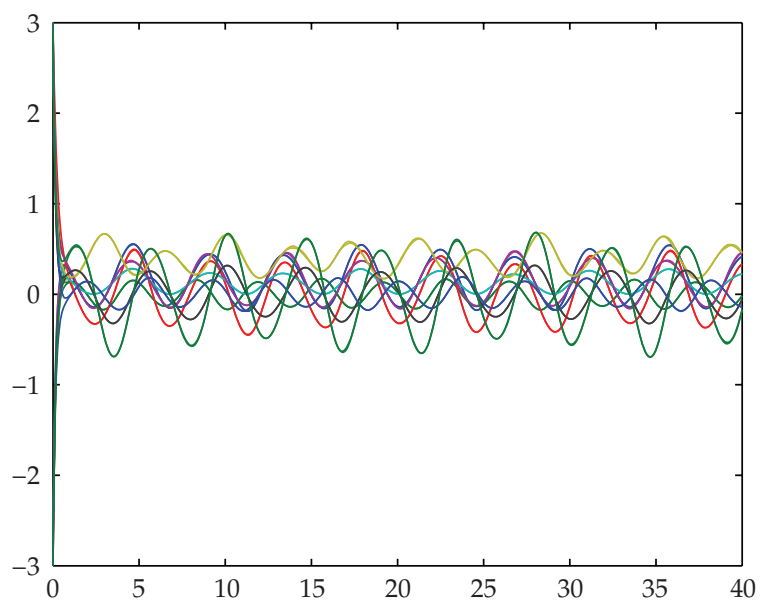

Figure 2: States of networks (5.1) with $t \in[0,40]$.

\section{Acknowledgments}

The authors would like to thank the editor Allan C Peterson and the reviewers for their valuable comments and constructive suggestions, which considerably improve the presentation of this paper. This work was jointly supported by the National Natural Science Foundation of China under Grant no. 60874088, the Foundation of Chinese Society for Electrical Engineering (2008), the China Postdoctoral Science Foundation under Grant no. 20070410300, 200801336, the Hunan Provincial Natural Science Foundation of China under Grant no. 07JJ4001, the Scientific Research Fund of Yunnan Provincial Education Department under Grant no. 07Y10085, and the Scientific Research Fund of Yunnan Province under Grant no. $2008 \mathrm{CD} 186$.

\section{References}

[1] A. Bouzerdoum and R. B. Pinter, "Shunting inhibitory cellular neural networks: derivation and stability analysis," IEEE Transactions on Circuits and Systems I, vol. 40, no. 3, pp. 215-221, 1993.

[2] A. Bouzerdoum and R. B. Pinter, "Analysis and analog implementation of directionally sensitive shunting inhibitory cellular neural networks," in Visual Information Processing: From Neurons to Chips, vol. 1473 of Proceedings of SPIE, pp. 29-38, 1991.

[3] A. Bouzerdoum and R. B. Pinter, "Nonlinear lateral inhibition applied to motion detection in the fly visual system," in Nonlinear Vision, R. B. Pinter and B. Nabet, Eds., pp. 423-450, CRC Press, Boca Raton, Fla, USA, 1992.

[4] K. Gopalsamy and X. Z. He, "Stability in asymmetric hopfield nets with transmission delays," Physica $D$, vol. 76, no. 4, pp. 344-358, 1994.

[5] Y. Li, C. Liu, and L. Zhu, "Global exponential stability of periodic solution of shunting inhibitory CNNs with delays," Physics Letters A, vol. 337, pp. 46-54, 2005.

[6] H. Meng and Y. Li, "New convergence behavior of shunting inhibitory cellular neural networks with time-varying coefficients," Applied Mathematics Letters, vol. 21, no. 7, pp. 717-721, 2008.

[7] B. Liu and L. Huang, "Existence and stability of almost periodic solutions for shunting inhibitory cellular neural networks with continuously distributed delays," Physics Letters A, vol. 349, no. 1-4, pp. 177-186, 2006.

[8] B. Liu and L. Huang, "Existence and stability of almost periodic solutions for shunting inhibitory cellular neural networks with time-varying delays," Chaos, Solitons and Fractals, vol. 31, no. 1, pp. 211-217, 2007. 
[9] Q. Zhou, B. Xiao, Y. Yu, and L. Peng, "Existence and exponential stability of almost periodic solutions for shunting inhibitory cellular neural networks with continuously distributed delays," Chaos, Solitons and Fractals, vol. 34, no. 3, pp. 860-866, 2007.

[10] A. Chen and J. Cao, "Almost periodic solution of shunting inhibitory CNNs with delays," Physics Letters A, vol. 298, no. 2-3, pp. 161-170, 2002.

[11] X. Huang and J. Cao, "Almost periodic solution of shunting inhibitory cellular neural networks with time-varying delay," Physics Letters A, vol. 314, no. 3, pp. 222-231, 2003.

[12] L. Chen and H. Zhao, "Global stability of almost periodic solution of shunting inhibitory cellular neural networks with variable coefficients," Chaos, Solitons and Fractals, vol. 35, no. 2, pp. 351-357, 2008.

[13] Y. Xia, J. Cao, and Z. Huang, "Existence and exponential stability of almost periodic solution for shunting inhibitory cellular neural networks with impulses," Chaos, Solitons and Fractals, vol. 34, no. 5, pp. 1599-1607, 2007.

[14] C. Ou, "Almost periodic solutions for shunting inhibitory cellular neural networks," Nonlinear Analysis: Real World Applications, vol. 10, no. 5, pp. 2652-2658, 2009.

[15] W. Zhao and H. Zhang, "On almost periodic solution of shunting inhibitory cellular neural networks with variable coefficients and time-varying delays," Nonlinear Analysis: Real World Applications, vol. 9, no. 5, pp. 2326-2336, 2008.

[16] J. Shao, L. Wang, and C. Ou, "Almost periodic solutions for shunting inhibitory cellular neural networks without global Lipschitz activity functions," Applied Mathematical Modelling, vol. 33, no. 6, pp. 2575-2581, 2009.

[17] H. Ding, J. Liang, and T. Xiao, "Existence of almost periodic solutions for SICNNs with time-varying delays," Physics Letters A, vol. 372, no. 33, pp. 5411-5416, 2008.

[18] B. Liu, "New convergence behavior of solutions to shunting inhibitory cellular neural networks with unbounded delays and time-varying coefficients," Applied Mathematical Modelling, vol. 33, no. 1, pp. 54-60, 2009.

[19] Y. Li and L. Huang, "Exponential convergence behavior of solutions to shunting inhibitory cellular neural networks with delays and time-varying coefficients," Mathematical and Computer Modelling, vol. 48, no. 3-4, pp. 499-504, 2008.

[20] Y. Li, H. Meng, and Q. Zhou, "Exponential convergence behavior of shunting inhibitory cellular neural networks with time-varying coefficients," Journal of Computational and Applied Mathematics, vol. 216, no. 1, pp. 164-169, 2008.

[21] J. Shao, L. Wang, and C. Ou, "Almost periodic solutions for shunting inhibitory cellular neural networks without global Lipschitz activity functions," Applied Mathematical Modelling, vol. 33, no. 6, pp. 2575-2581, 2009.

[22] C. Y. He, Almost Periodic Differential Equation, Higher Education, Beijing, China, 1992.

[23] E. F. Beckenbach and R. Bellman, Inequalities, Springer, Berlin, Germany, 1965. 


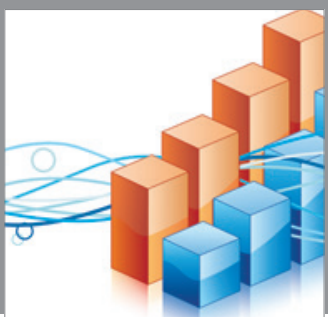

Advances in

Operations Research

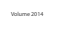

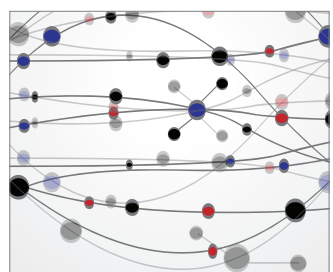

\section{The Scientific} World Journal
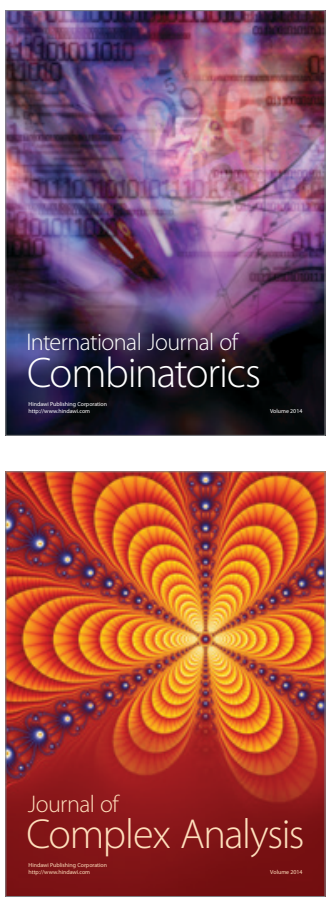

International Journal of

Mathematics and

Mathematical

Sciences
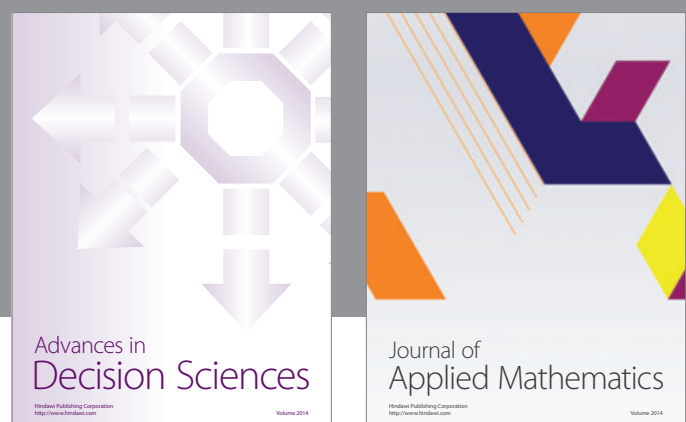

Journal of

Applied Mathematics
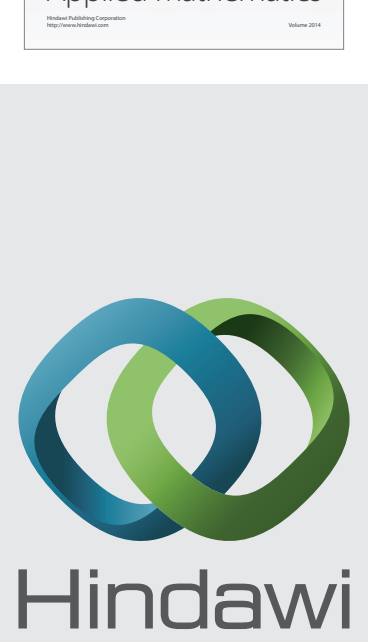

Submit your manuscripts at http://www.hindawi.com
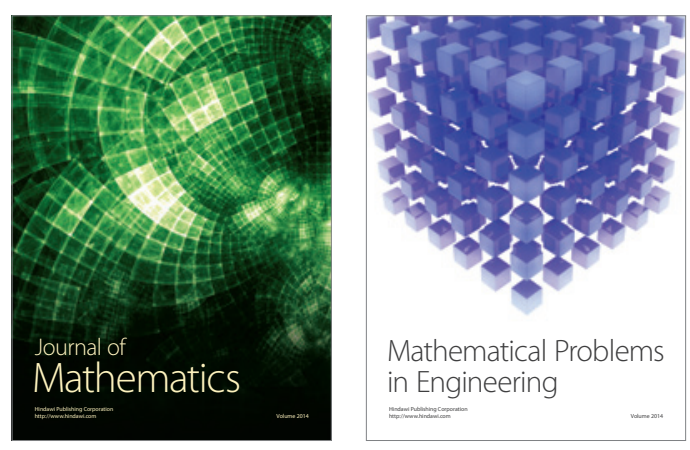

Mathematical Problems in Engineering
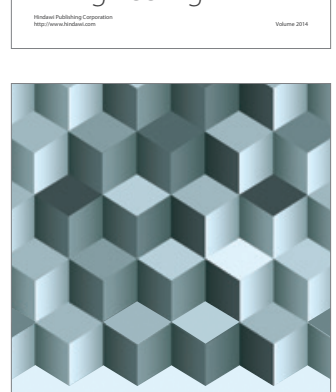

Journal of

Function Spaces
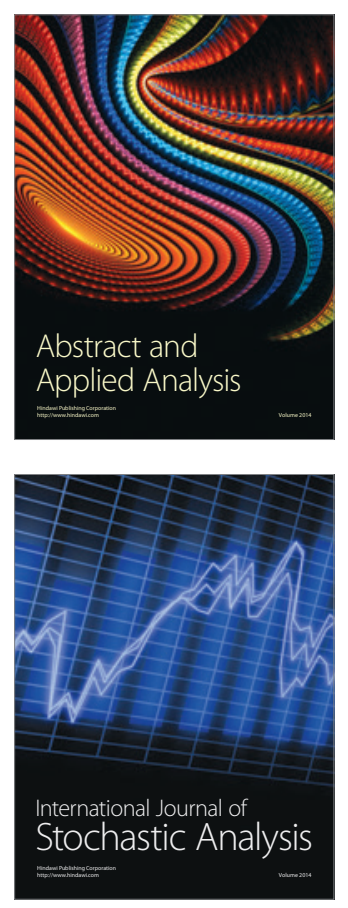

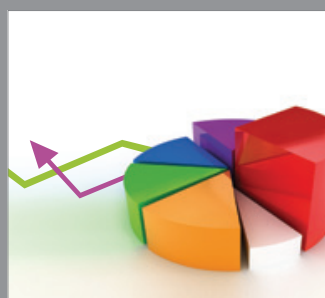

ournal of

Probability and Statistics

Promensencen
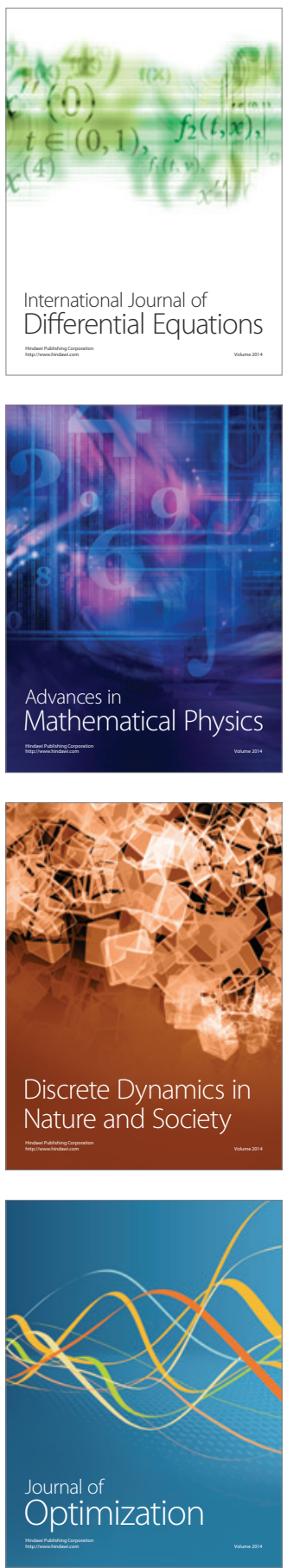